\title{
Welcome to the 15th volume of Future Microbiology
}

\author{
Atiya Henry*,1 \\ ${ }^{1}$ Future Science Group, Unitec House, 2 Albert Place, London, N31QB, UK \\ *Author for correspondence: a.henry@futuremedicine.com
}

Keywords: antimicrobial resistance $\bullet$ Candida auris $\bullet$ drug repurposing • infectious diseases $\bullet$ pathogen-associated acute encephalitis syndrome $\bullet$ poliopolis $\bullet$ sarecycline $\bullet$ Staphylococcus aureus $\bullet$ microbiology

To our readers, we would like to wish you a very happy new year from all of us here at Future Medicine and introduce you to the 15th volume of Future Microbiology.

I would like to begin 2020 with a thank you to our readers, esteemed Editorial Board, peer reviewers and contributing authors for their continued support. We look forward to working with you all in the forthcoming year! This foreword presents our content highlights of 2019, alongside some exciting future prospects for the journal, in the year ahead.

\section{Content highlights}

The year 2019 was another successful year for Future Microbiology. The journal fulfilled its purpose by publishing exciting current research from the experts in the field, comprising a varied spread of topics representative of the fast-evolving world of microbiology.

The top read review and the article with the highest number of reads for 2019 (as of January 2020) was 'Sarecycline: a narrow spectrum tetracycline for the treatment of moderate-to-severe acne vulgaris' by Moore et al. [1]. In this article the authors gave an insight into the pharmacology and clinical trial performance of Sarecycline, a US FDA-approved oral antibiotic used to treat moderate-to-severe acne vulgaris.

The second highest-read article overall was an editorial entitled 'The impact of infectious disease in war time: a look back at WW1' written by Hugh Pennington [2]. The short piece provides an interesting summary of the infectious diseases soldiers faced in WW1, how they were managed and how the outbreaks progressed.

Another highly popular paper was a special report entitled 'Reporting elevated vancomycin minimum inhibitory concentration in methicillin-resistant Staphylococcus aureus: consensus by an International Working Group' written by Wilcox et al. [3]. In this piece the authors' provide their recommendations on the most suitable methodology to determine the minimum inhibitory concentration of vancomycin in methicillin-resistant $S$. aureus.

Volume 14's most popular research article was 'Novel multiplex real-time quantitative PCR detecting system approach for direct detection of Candida auris and its relatives in spiked serum samples' written by Arastehfar et al. [4]. Here, the team developed a highly specific and sensitive multiplex qPCR assay to detect C. auris, Candida haemulonii, Candida duobushaemulonii and Candida pseudohaemulonii from clinical samples.

\section{Readership demographics}

It is always interesting to consider the demography of our content's impact, as it continues to change with the increasing growth of Future Microbiology. At 31\%, this year Asia remains our largest readership proportion. Our second and third highest readerships belonged to the USA (29\%) and Europe (28\%) (Figure 1). This represents a growth of $5 \%$ for the USA and $2 \%$ for Europe since last year. We expect to see continued growth in emerging scientific communities, in hopes of reaching a truly globalized audience.

\section{Top Altmetric articles}

We have been working with Altmetric since 2015 to help visualize where the articles we publish are having online impact. A special acknowledgment goes out to this year's top three articles, which all scored in the top $25 \%$ of all research outputs scored by Altmetric, in Future Microbiology.

Future $\because \cdots$ Medicine 


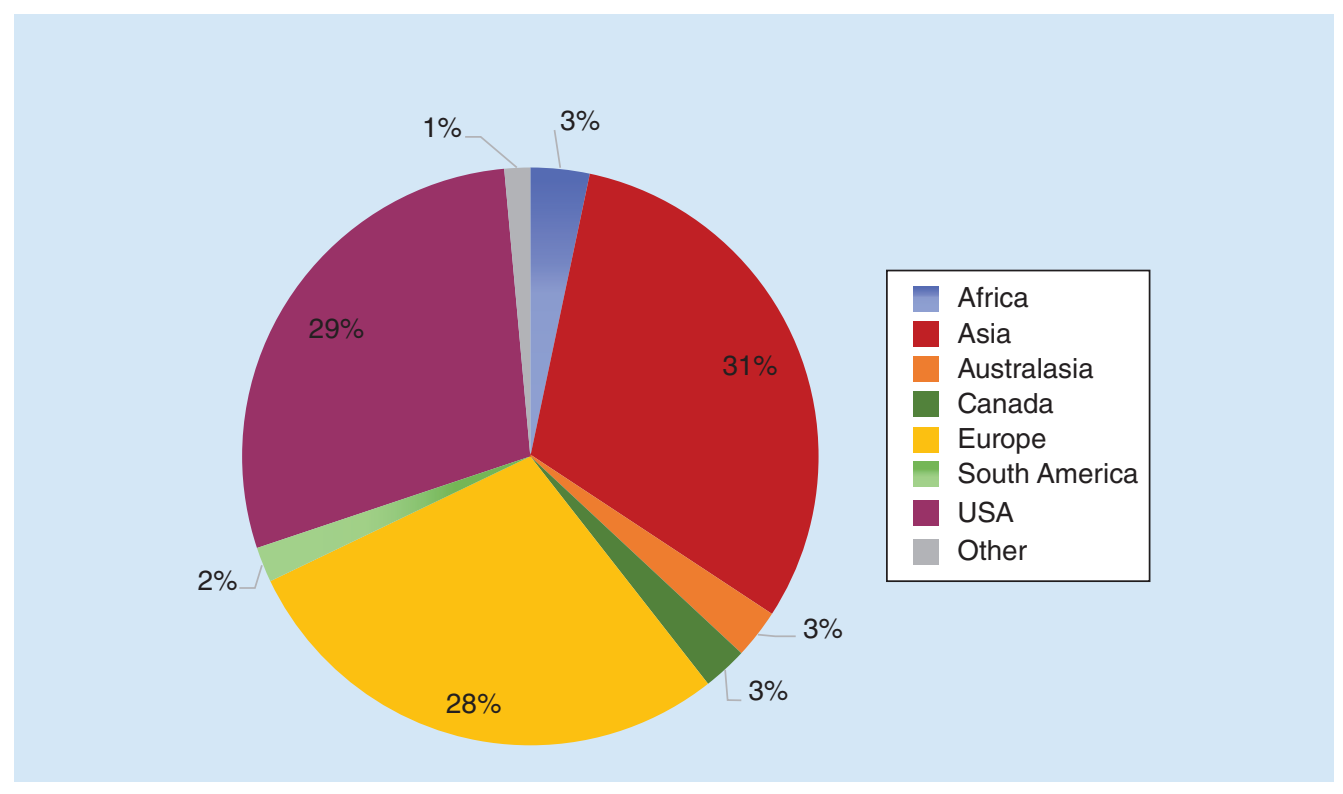

Figure 1. Future Microbiology readership demographic 2019.

- The highest scoring piece was a special report entitled 'Poliopolis: pushing boundaries of scientific innovations for disease eradication' [5] which was in the top 5\% of all research outputs scored by Altmetric. This piece was picked up by 13 news outlets;

- A perspective entitled 'Combating resistance while maintaining innovation: the future of antimicrobial stewardship' [6] was at second and in the top $5 \%$ of all research outputs scored by Altmetric;

- Finally, the editorial article 'Pathogen-associated acute encephalitis syndrome: therapeutics and management' came third [7].

\section{Social media}

Future Microbiology remains active across social media and you can follow our latest updates on Twitter (@fsgfmb) and LinkedIn [8]. Across these platforms, we discuss the most recent research in the field of microbiology, and the latest news, journal highlights and newly published articles. Through these mediums, we continue to enjoy engaging with our readers and experts in the field.

\section{Infectious diseases hub}

Future Microbiology is proudly partnered with Infectious Diseases Hub [9], a free-access online resource that provides a platform to allow quick access to the latest, essential research to its members on all aspects of virology and microbiology, from the bench to the bedside. Breaking news, exclusive features, interviews, peer-reviewed articles and more are covered in Infectious Diseases Hub, provided by Future Science Group [10]. Additionally, three articles are nominated each month from the corresponding issue to feature on Infectious Disease Hub, at the discretion of the editor. One popular example that considered a 'hot topic' in microbiology of antimicrobial resistance was a 'peek behind the paper' piece entitled 'Sidharth Chopra on drug repurposing to tackle antimicrobial resistance' with lead author, Sidharth Chopra [11,12]. The full review was offered as free access to all members of Infectious Disease Hub, in addition to this exclusive interview where the value and limitations of drug repurposing are discussed, with perspectives for the future. This affiliation has allowed the content of Future Microbiology to have wider global reach and increased dissemination and influence through a multitude of platforms.

\section{Conference attendance}

Following previous years, members of the Future Microbiology department attended a number of conferences, meetings and events around the world allowing us to meet our readers, authors, Editorial Board members and collaborators, as well as keep up-to-date with the field and ensure full coverage of all the latest and ground-breaking research. In 2019, we attended the following events: 
- Microbiology Society Annual Conference (8-11 April, Belfast, UK);

- The European Congress of Clinical Microbiology and Infectious Diseases (ECCMID; 13-16 April, Amsterdam, Netherlands);

- American Society for Microbiology (ASM) Microbe (20-24 June, San Francisco, CA, USA).

The Future Microbiology team looks forward to attending more conferences to continue further journal development. To find out which events we will be attending in 2020, please follow the journal on Twitter [13], join our LinkedIn group [8] or become a member of IDHub for free [9].

\section{Conclusion}

As always, any feedback the microbiology community wishes to share is appreciated, whether this be recommendations of up-and-coming areas you think deserve coverage or advice on the future direction of our content, corresponding to changing directions in the field. We welcome unsolicited research, review, editorial and opinion article proposals, among others and would be delighted to hear from you if you are interested in submitting to the journal. We are especially interested in submissions covering key aspects of microbiology, including:

- The molecular basis of microbial diseases;

- Microbe-host interactions;

- Current and future therapeutic and diagnostic approaches;

- The use of microbes in the treatment of disease and genetic engineering;

- Newly approved antimicrobial agents;

- Pharmacoeconomics and cost-benefit issues in microbiology;

- New and re-emerging microbes impacting human health;

- Microbial genome research and the implications to higher organisms;

- Microbes and cancer;

- Epidemiologic studies and trends;

- Drug resistance (e.g., MRSA), and potential methods to overcome this;

- Microbes in bioterrorism.

\section{Financial \& competing interests disclosure}

The author is an employee of Future Science Group. The author has no other relevant affiliations or financial involvement with any organization or entity with a financial interest in or financial conflict with the subject matter or materials discussed in the manuscript apart from those disclosed.

No writing assistance was utilized in the production of this manuscript.

\section{References}

1. Moore AY, Charles JE, Moore S. Sarecycline: a narrow spectrum tetracycline for the treatment of moderate-to-severe acne vulgaris. Future Microbiol. 14(14), 1235-1242 (2019).

2. Pennington H. The impact of infectious disease in war time: a look back at WW1. Future Microbiol. 14(3), 165-168 (2019).

3. Wilcox M, Al-Obeid S, Gales A et al. Reporting elevated vancomycin minimum inhibitory concentration in methicillin-resistant Staphylococcus aureus: consensus by an International Working Group. Future Microbiol. 14(4), 345-352 (2019).

4. Arastehfar A, Fang W, Daneshnia F et al. Novel multiplex real-time quantitative PCR detecting system approach for direct detection of Candida auris and its relatives in spiked serum samples. Future Microbiol. 14(1), 33-45 (2019).

5. Van Damme P, Coster ID, Bandyopadhyay AS et al. Poliopolis: pushing boundaries of scientific innovations for disease eradication. Future Microbiol. 14(15), 1321-1330 (2019).

6. Vickers RJ, Bassetti M, Clancy CJ et al. Combating resistance while maintaining innovation: the future of antimicrobial stewardship. Future Microbiol. 14(15), 1331-1341 (2019).

7. Saxena SK, Kumar S, Maurya VK. Pathogen-associated acute encephalitis syndrome: therapeutics and management. Future Microbiol. 14(4), 259-262 (2019).

8. Linkedin group: Future Science Group Microbiology. www.linkedin.com/groups/8506116.

9. Infectious Diseases Hub. www.id-hub.com.

10. Future Science Group. www.future-science-group.com/. 
11. A peek behind the paper - Sidharth Chopra on drug repurposing to tackle antimicrobial resistance. ID Hub (2019). www.id-hub.com/2019/09/05/peek-behind-paper-sidharth-chopra-drug-repurposing-tackle-antimicrobial-resistance/.

12. Kaul G, Shukla M, Dasgupta A, Chopra S. Update on drug-repurposing: is it useful for tackling antimicrobial resistance? Future Microbiol. 14(10), 829-831 (2019).

13. Future Microbiol. Twitter. https://twitter.com/fsgf mb. 